\title{
Algunos retos y propuestas en la conceptualización, evaluación e intervención del Trastorno Específico del Lenguaje (TEL)
}

\author{
Some challenges and proposals in the \\ conceptualization, assessment and intervention of \\ Specific Language Impairment (SLI)
}

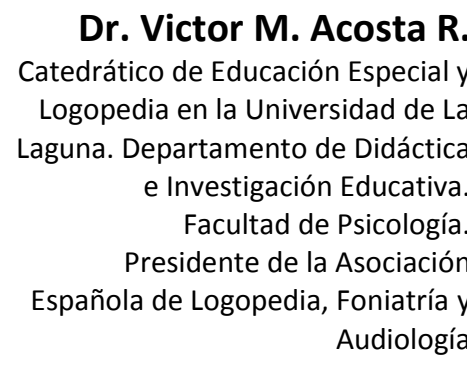

Contacto con el autor: Dr. Victor M. Acosta R. C/. EL Acebiño, no1, Urbanización Jardín del Sol. 38350 Tacoronte (S/C de Tenerife-Islas Canarias). España. Tel: (34922) 571737 Te,:(34-922) 319025 Correo-e:vacosta@ull.es

Financiación: Ministerio de Economía y Competitividad del Gobierno de España. Proyecto de Investigación Funciones ejecutivas y lenguaje en alumnado con TEL. Un modelo de evaluación e intervención con bases psicolingüística y neuropsicológica. Referencia EDU2011-27789

\section{RESUMEN}

El presente trabajo tiene como objetivo principal reflexionar sobre algunos tópicos actuales en torno al Trastorno Específico del Lenguaje (TEL), fruto de nuestro trabajo investigador de los últimos años y de los nuevos retos que hemos afrontado. En primer lugar, se revisan aquellos aspectos cruciales en la conceptualización de este trastorno. En segundo lugar, se analiza la problemática alrededor de su evaluación y se hacen propuestas de instrumentos a utilizar para una identificación temprana. Finalmente, se sugiere un modelo de trabajo basado en diferentes niveles de intervención, en la colaboración profesional y en la combinación de procedimientos y estrategias.

Palabras claves: trastorno específico del lenguaje, evaluación, intervención.

\section{ABSTRACT}

The main objective of this paper is to reflect on some current issues about the Specific Language Impairment (SLI), as a result of our research work carried out lately and the new challenges we have faced up to. Firstly, we go through the key aspects in the conceptualization of this impairment. Secondly, we analyze the problems associated with assessment and we proposed some instruments to be used for early identification. Finally, we suggest a working approach based on different levels of intervention, on professional collaboration and on the combination of procedures and strategies.

Key words: specific language impairment, assessment, intervention. 


\section{Introducción}

El término Trastorno Específico del Lenguaje (TEL) suele utilizarse para referirse a un tipo de niños que presenta serios problemas en su lenguaje y una aparente ausencia de otras dificultades tales como déficit intelectual no verbal, autismo, trastorno sensorial, daño neurológico o deprivación sociocultural ${ }^{1}$. Al tratarse de un trastorno dinámico no resulta sencilla su identificación temprana, por lo que se ha recurrido a una enorme variabilidad de criterios $^{2}$. En primer lugar, se sugiere establecer un corte a partir de una puntuación en al menos dos tests de lenguaje de -1,25 desviaciones estándar o más baja ${ }^{3,4,5}$. En segundo lugar, se suele acudir también a diversos marcadores clínicos. Entre éstos, se han señalado de modo insistente dos problemas que tienen estos niños para, por un lado, usar de manera adecuada determinadas formas gramaticales, entre las que sobresale el empleo del tiempo pasado en los verbos ${ }^{6,7,8}$ y por otro lado, para la realización correcta de tareas de repetición de pseudopalabras, es decir, la imitación de palabras sin sentido que varían en su longitud silábica y en su complejidad fonológica $^{9,10}$. En tercer lugar, y debido a hallazgos recientes, se ha revelado que los niños con TEL tienen mayores problemas que sus pares para el aprendizaje de objetivos específicos del lenguaje como por ejemplo habilidades conversacionales, narrativas o académicas ${ }^{11,12,13,14}$. Por último, se han manifestado una serie de factores de riesgo, entre los cuales cabría señalar, la presencia de trastornos del lenguaje en los progenitores, el sexo (mayor frecuencia entre los niños), el orden de nacimiento (notable riesgo para los que nacen más tarde), o el nivel educativo de los padres ${ }^{15}$.

Asimismo, en los últimos años, se está considerando otro grupo de variables no estrictamente lingüísticas, muchas de las cuales estarían posiblemente vinculadas con el TEL. Por ejemplo, se han destacado algunos problemas neurológicos (alteraciones del córtex frontal y de los ganglios basales ${ }^{16}$ ), cognitivos y de procesamiento de la información (memoria de trabajo verbal, procesamiento temporal de estímulos auditivos o de procesamiento fonológico, razonamiento, según Montgomery ${ }^{17}$ ), genéticos (antecedentes familiares con dificultades de lenguaje $\mathrm{e}^{18,19}$ ).

\section{Características del lenguaje en los TEL.}

En la literatura científica se ha descrito a los TEL como un grupo muy heterogéneo. Por un lado, en las clasificaciones internacionales más utilizadas (ver por ejemplo, el DSM-IV y el ICD-10) se establece una diferencia entre aquellos que presentan problemas tanto en la comprensión como en la expresión del lenguaje, de los que muestran dificultades únicamente expresivas. Por otro lado, se describe a los TEL como un trastorno muy singular en el que concurren un buen número de peculiaridades lingüísticas.

En efecto, muchos niños con TEL exhiben en sus primeros años de vida, auténticas dificultades para interaccionar con otras personas de su ambiente y poner en juego habilidades de reciprocidad, atención conjunta y gestos simbólicos; en esta etapa inicial también se ha podido detectar un cierto 
retraso en la producción de balbuceo canónico, de patrones de fonología temprana y de determinadas consonantes. En trabajos recientes, por ejemplo Bosch $^{20}$ (2012), se señala igualmente una alta correlación entre aquellos niños que no están extrayendo a tiempo la información relativa a los sonidos propios de la lengua que están adquiriendo y un posterior retraso en la adquisición de los niveles más avanzados del desarrollo del habla/lenguaje. Esta misma autora sugiere además el posible valor predictivo de la habilidad temprana para segmentar el habla e identificar palabras, antes de los 12 meses de edad, y medidas de crecimiento léxico y gramatical en edades más avanzadas (4 a 6 años). De todo ello se desprende una cierta relación entre los problemas del habla y los trastornos del lenguaje, concretándose en un desarrollo más tardío del vocabulario y de la morfosintaxis, tal y como se sugiere también en los trabajos de Acosta, León y Ramos ${ }^{21}$ o de Pavez, Maggiolo y Coloma ${ }^{22}$.

Como ya se expresó anteriormente, el deterioro del componente morfosintáctico del lenguaje se considera la característica más común de los niños con TEL; al parecer suelen presentar una cierta ceguera para abstraer las reglas implícitas que gobiernan la gramática de la lengua ${ }^{23}$. Ello se pone de manifiesto en la presencia de una limitada Longitud Media de Enunciados (LME), del uso predominante de frases simples y de una menor aparición de frases compuestas, especialmente de las subordinadas, o de una mayor dificultad para el empleo de artículos y pronombres.

Los problemas pragmáticos y de discurso también han sido descritos en niños con TEL. De hecho Bishop ${ }^{24}$, los vincula con un subgrupo particular de TEL, y más concretamente con el trastorno semántico-pragmático. Este asunto no es una cuestión menor ya que ha llevado a establecer una cierta relación entre este trastorno y los problemas de niños con trastornos leves del espectro autista (TEA). En efecto, aunque a primera vista los desórdenes de estos dos tipos de niños se pueden identificar como distintos, la presentación clínica puede ser compleja y no necesariamente clara para el diagnóstico diferencial. Particularmente, se establece la existencia de marcadores comunes (concretamente comportamientos comunicativos similares) entre estos dos tipos de dificultades del desarrollo ${ }^{2}$. Con todo, hay algunas señales de alerta que ayudan al diagnóstico diferencial; así, la pérdida del lenguaje, una ausencia en la conducta de mostrar o indicar, una falta del uso compartido de intereses, una inexistencia de la mirada apropiada, la carencia de respuesta a su nombre o la presencia de movimientos repetitivos del cuerpo o con determinados objetos, son conductas más propias de los TEA que de los TEL, lo que hace que los primeros tengan siempre presente una triada de problemas que afectan a la comunicación, a la interacción social y al repertorio conductual ${ }^{25 .}$.

Por último, se han encontrado complicaciones importantes en la narrativa de los niños con TEL. Las habilidades narrativas constituyen un aspecto central a tener en cuenta en el estudio de este trastorno. Por un lado nos ofrecen información general sobre el desarrollo lingüístico; por otro, nos conectan con diversas habilidades consideradas centrales en el proceso de alfabetización. Por esta 
razón, existe un gran número de investigadores que recomiendan dedicar un tiempo a examinar en profundidad la narrativa de estos niños, especialmente en edades tempranas, como medida medular a través de la cual poder inferir un perfil general de su desarrollo ${ }^{26}$.

Como ya hemos señalado en un trabajo anterior $^{27}$, el estudio de la narrativa en niños con TEL debe partir de un buen esquema de análisis. En líneas generales, se necesita confeccionar una doble exploración. En primer lugar, la obtención de datos procedentes de un análisis microestructural y que atiende a los rasgos gramaticales y semánticos de la narración. Así, medidas de cohesión que aportan información sobre las características internas de las narraciones y de cómo se articulan sus diferentes elementos de manera significativa, para proporcionar información acerca de su calidad y complejidad. Algunos de estos marcadores cohesivos lingüísticos tienen que ver con el uso de pronombres, conjunciones, conectores adverbiales, artículos definidos e indefinidos, etc. En segundo lugar, debe procederse a un análisis macroestructural, consistente en el examen de la organización general y de la estructura episódica de la narrativa; más concretamente se señalan los patrones estructurales y sus propiedades como, por ejemplo, la presentación, el episodio y el final ${ }^{22}$.

Pues bien, hay evidencia de que los niños con TEL presentan dificultades con sus habilidades narrativas $^{22,27,28,29,30,31,32}$. Se ha constatado que las narraciones de los niños con TEL tienden a contener menos información y una menor madurez lingüística cuando se las compara con las realizadas por niños con desarrollo típico. Probablemente las limitaciones lingüísticas de los TEL provocan una incapacidad real para expresar distintos fragmentos de información. La situación anterior se agrava si se tiene en cuenta la presencia de una doble dificultad en los TEL. En primer lugar, para relacionar la información nueva con el conocimiento previo; $y$, en segundo lugar, para manejar ciertas palabras abstractas, tales como términos temporales y deícticos, así como para recuperar rápidamente determinadas palabras que suelen sustituir por otras de menor contenido.

\section{La evaluación en los TEL: sujetos y contextos}

Cuando se habla de evaluación hay que hacer mención a dos grandes ámbitos. En primer lugar al estudio del individuo como procesador del input que recibe en los contextos en los que se desenvuelve. En segundo lugar es importante conocer cómo la experiencia social y las herramientas socioculturales propician y guían el aprendizaje en general, y la adquisición del lenguaje, en particular. Ahora cobran mucho valor un conjunto de nociones, entre las que cabría destacar las de contexto, prácticas educativas, adaptación, apoyo y recursos materiales.

\section{Sujetos:}

Existen serias dificultades para evaluar a los niños con TEL debido, entre otras razones, a la gran dispersión y heterogeneidad de instrumentos. Tal y como nos recuerda Mendoza (2011) ${ }^{33}$, la generación de protocolos de evaluación científicamente validados sería un objetivo necesario para reconocer adecuadamente a los niños con TEL y para adoptar 
las medidas preventivas necesarias desde los años de educación infantil (0-6 años). Esta misma investigadora refiere al sistema EpiSLI como uno de los primeros métodos de screening para identificar a los $\mathrm{TEL}^{5}$ y que fue utilizado en un amplio estudio epidemiológico de niños de habla inglesa. En este sistema se establecen las áreas lingüísticas específicas (vocabulario, gramática y narrativa), en las modalidades de comprensión y producción, que pueden ser indicadoras de un TEL, y que Mendoza ${ }^{33}$ ha actualizado convenientemente, con la introducción de algunos marcadores psicolingüísticos novedosos (discriminación fonológica, repetición de pseudopalabras, conciencia fonológica, fluidez léxica, comprensión y producción gramatical, bootstrapping gramatical, etc.).

En un trabajo reciente de Carballo $^{34}$ se insiste en la importancia de una identificación temprana del TEL, mediante el uso de marcadores que predicen el posterior desarrollo del lenguaje, sugiriéndonos de esta manera el empleo del Cuestionario Parental: CSBS-DP (Communication and Symbolic Behavior Scales: Developmental Profile ${ }^{35}$ ), o de listas de prueba o de control (checklist), como la Lista de Comunicación y Desarrollo del Lenguaje ${ }^{35}$; la adaptación española del Inventario de Desarrollo Comunicativo MacArthur ${ }^{36}$, la CCC-2 (Children's Communication Checklist) de Bishop ${ }^{24}$, o las Escalas de rangos observacionales del CELF-4 ${ }^{37}$.

El grupo de investigación en dificultades de lenguaje Acentejo, vinculado a la Universidad de La Laguna (Islas Canarias, España) ha utilizado una serie de instrumentos para la identificación y la evaluación tempranas de niños con TEL, tal y como se recoge en la tabla I (para una mayor revisión puede consultarse los trabajos de Acosta, Moreno y Axpe ${ }^{11,29}$ ).

\section{Contextos:}

Junto con la evaluación de las habilidades del alumnado con TEL, también deberían estudiarse los ambientes en los que se ejecutan las prácticas, y más concretamente los contextos aula y hogar.

Para evaluar de forma adecuada el contexto del aula, se sugiere recurrir a la Guía de Observación ELLCO-PreK de Smith, Brady y Anastasopoulos ${ }^{38}$. Se trata de un instrumento de observación que contiene un total de 19 ítems, organizados en cinco grandes secciones. La sección I, Estructura de la clase, contiene cuatro ítems para la organización general del aula y los contenidos que en ella se imparten, la disponibilidad de materiales y el uso que hacen los niños de ellos, el manejo de las prácticas docentes, y el papel de los distintos profesionales que intervienen. La sección II, Curriculum, consta de tres ítems que buscan información sobre las estrategias de enseñanza, las oportunidades de aprendizaje ofrecidas al alumnado para que participe y tome la iniciativa, y la actitud de los profesionales hacia la diversidad. La sección III, el Lenguaje en el Aula, consta de cuatro ítems que intentan detectar el clima del discurso utilizado, las oportunidades ofrecidas para extender las conversaciones, la importancia dada al vocabulario, y el papel concedido al trabajo en conciencia fonológica. La sección IV, Libros y lectura con libros, contiene cinco ítems, que buscan información acerca de la posible existencia en el aula de una zona destinada a la organización y el uso de los libros, las características de los libros disponibles, 
la presencia y el uso de libros en las distintas áreas curriculares, y la calidad y la frecuencia de la lectura de libros. La sección V, Material impreso y escritura inicial, incorpora tres ítems focalizados en la detección de los materiales para la escritura, las oportunidades que se ofrecen para que el niño pueda conseguir una conciencia adecuada del material escrito y la variedad de propósitos para los que la lectura puede ser empleada, y el uso en el aula de diferentes tipos de material impreso.

Por otra parte, existen diferentes instrumentos para evaluar el contexto familiar. En primer lugar, se puede aplicar el Cuestionario sobre Prácticas de Alfabetización Temprana ${ }^{39}$, con el objetivo de obtener información sobre el uso del lenguaje oral y la lectura inicial en el hogar, los recursos disponibles, el reconocimiento de material escrito, el trabajo en conciencia fonológica y en escritura emergente.

En segundo lugar, conviene registrar una situación de lectura de un cuento conocido (p.e. Los tres cerditos, La pequeña oruga glotona), que una vez transcrita se puede analizar mediante el empleo del Inventario de Lectura Interactiva $(A C I R I)$ de DebruinParecki $^{40}$, cuyo uso permite conocer si los padres y los niños muestran o no un estilo interactivo de lectura caracterizado por facilitar la atención del niño hacia el texto (categoría I), promocionar la lectura interactiva y apoyar la comprensión (categoría II) y usar estrategias para andamiar el aprendizaje del niño (usar señales visuales, formular preguntas que establezcan predicciones, ayudar a recordar la información y ofrecer ideas acerca de la historia, constituyen la categoría III).
Para una revisión amplia del uso de las herramientas anteriores en niños con TEL puede consultarse el trabajo de Acosta, Moreno y Axpe ${ }^{41}$.

\section{La intervención en los TEL}

Una revisión de la literatura nos ofrece diversas aproximaciones en la intervención sobre niños con $\mathrm{TEL}^{42}$. Frecuentemente se recurre a una clasificación clásica que establece una diferencia entre modelos naturales (que emplean técnicas de enseñanza menos intrusivas) y modelos directos y focalizados (que usan procedimientos más precisos y estructurados).

En la misma dirección, algunos autores distinguen entre métodos funcionales y formales; en los primeros, los objetivos de intervención se ajustan a la propia iniciativa del niño, cuidándose mucho la organización de los contextos y los intercambios comunicativos niño-adulto; mientras que en los segundos, se fomenta el trabajo individual, en situaciones mucho más restringidas, siguiendo una secuencia que comienza por la comprensión y continúa con la imitación, la expresión controlada y la generalización $^{43,44}$. Finalmente, y siempre en la misma línea argumental, nos encontramos con la propuesta de Mendoza ${ }^{45}$, quien distingue diferentes enfoques que se extienden a lo largo de un continuo que oscila entre los planteamientos altamente estructurados y los procedimientos interactivos (en el centro quedarían los que apoyan el uso funcional del lenguaje en las interacciones, denominados modelos de intervención en el medio). 
Tabla I. Instrumentos utilizados para la detección y la evaluación de niños con TEL.

\begin{tabular}{|c|c|}
\hline Pruebas & Habilidad/Competencia \\
\hline Escala de Desarrollo Cognitivo General (McCarthy, 1976) & Desarrollo cognitivo general \\
\hline Prueba de Lenguaje PLS-4 (Zimmerman, Steiner y Pond, 2004) & Lenguaje expresivo y comprensivo \\
\hline $\begin{array}{l}\text { Prueba del desarrollo inicial del lenguaje-Español (Hresko, Reid y } \\
\text { Hamill, 2007) }\end{array}$ & Lenguaje expresivo y comprensivo \\
\hline CELF-4 (Semel, Wiig y Secord, 2004) & Lenguaje expresivo y comprensivo \\
\hline Prueba de Discriminación Auditiva del A-RE-HA (Aguilar y Serra, 2003) & Percepción del habla \\
\hline $\begin{array}{l}\text { Integración auditiva (ITPA, Kirk, McCarty y Kirk,2005) y Registro } \\
\text { Fonológico Inducido (RFI) (Juárez y Monfort, 1996) }\end{array}$ & Programación fonológica \\
\hline $\begin{array}{l}\text { Pseudopalabras (Aguado, Cuetos, Domezaín y Pascual., 2006); } \\
\text { Imitación de frases (WPPSI, Wechsler, 1993) }\end{array}$ & Memoria de trabajo \\
\hline Peabody (Dunn, Padilla, Lugo y Dunn, 1986) & Identificación léxica \\
\hline Semejanzas y Vocabulario (WPPSI, Wechsler, 1993) & Semántica: expresión \\
\hline $\begin{array}{l}\text { Asociación Visual y Asociación Auditiva (ITPA, Kirk, McCarty y Kirk, } \\
\text { 2005) }\end{array}$ & Semántica: relación conceptual e inferencias \\
\hline Evaluación de Guiones (Pavez, Coloma y Maggiolo, 2008) & Desarrollo narrativo temprano \\
\hline $\begin{array}{l}\text { Contar y recontar cuentos con material visual. Responder a preguntas } \\
\text { literales e inferenciales. Cuentos utilizados: Buenas noches, gorila; } \\
\text { Rana, ¿dónde estás? }\end{array}$ & Producción y comprensión narrativas \\
\hline Conciencia fonológica (Jiménez y Ortiz,1995) & Conciencia fonológica \\
\hline Procesamiento fonológico-PROFON (Lara, Aguilar y Serra, 2007) & $\begin{array}{l}\text { Conciencia fonológica (niveles silábicos, } \\
\text { intrasilábico y fonémico). Denominación } \\
\text { fonológica. Memoria fonológica. Conocimiento } \\
\text { de las letras. Asociación fonema-grafema }\end{array}$ \\
\hline Análisis morfo-léxico & $\begin{array}{l}\text { Índice de diversidad léxico (IDL) } \\
\text { Longitud media de enunciados (LME) }\end{array}$ \\
\hline
\end{tabular}

Los modelos altamente estructurados siguen los planteamientos del conductismo y apelan al uso de procedimientos como la imitación, el moldeamiento, el desvanecimiento y la generalización (un ejemplo de lo anterior se pone en práctica cuando se pretenden conseguir objetivos relacionados con habilidades $\operatorname{articulatorias~}^{46}$ o morfosintácticas ${ }^{47}$, mientras que los modelos interactivos persiguen facilitar la comunicación social por medio del modelado interactivo; se ha señalado que pueden conseguirse habilidades lingüísticas concretas como por ejemplo facilitar la toma de turnos, participar en actividades narrativas, incrementar las oportunidades para el uso de lenguaje descontexualizado, aumentar el vocabulario o desarrollar habilidades de lectura temprana $^{48}$. Los enfoques situados en el medio del continuo incorporan una serie de procedimientos híbridos ya que recurren a técnicas conductistas pero aplicadas en situaciones de discurso conversacional (por ejemplo, la terapia basada en scripts; el uso de la conversación o de la narración para conseguir objetivos lingüísticos ${ }^{49,50}$ ). En cualquier caso, hay pocos datos empíricos que hablen de la efectividad y de la eficacia en la intervención global en los TEL y sus implicaciones para su desarrollo emocional, académico y social ${ }^{1}$. La mayor parte de los trabajos se han centrado en aspectos muy concretos, como la adquisición de preposiciones ${ }^{51}$, de determinadas formas morfosintácticas ${ }^{52}$, de pronombres personales $^{53}$, de vocabulario ${ }^{54}$ o de conciencia fonológica $^{55}$; con todo, como nos recuerda Mendoza $^{56}$, se sigue necesitando un mayor volumen 
de investigación aplicada que ofrezca evidencia empírica sobre la eficacia y la eficiencia de las distintas perspectivas de intervención en los TEL.

Precisamente nuestro interés en los últimos años ha sido poner en práctica modelos de trabajo de naturaleza educativa y fonoaudiológica dirigidos a conseguir un mayor desarrollo, aprendizaje e inclusión de alumnado con TEL, entre los 4 y los 8 años de edad ${ }^{57,58}$. Para tal fin hemos diseñado una propuesta de intervención sustentada sobre dos grandes pilares. El primero ha cuidado minuciosamente los contextos en los que se interviene, las variedades de los agrupamientos, el tipo y el grado de apoyo que se brinda, la colaboración entre profesionales, y una modalidad de evaluación claramente dinámica. El segundo ha conectado el diseño de objetivos de intervención con la frecuencia e intensidad de las sesiones, las estrategias (vertical, horizontal y/o cíclica), los procedimientos, las actividades y los materiales. Una representación de todo este proceso se muestra en la figura 1.

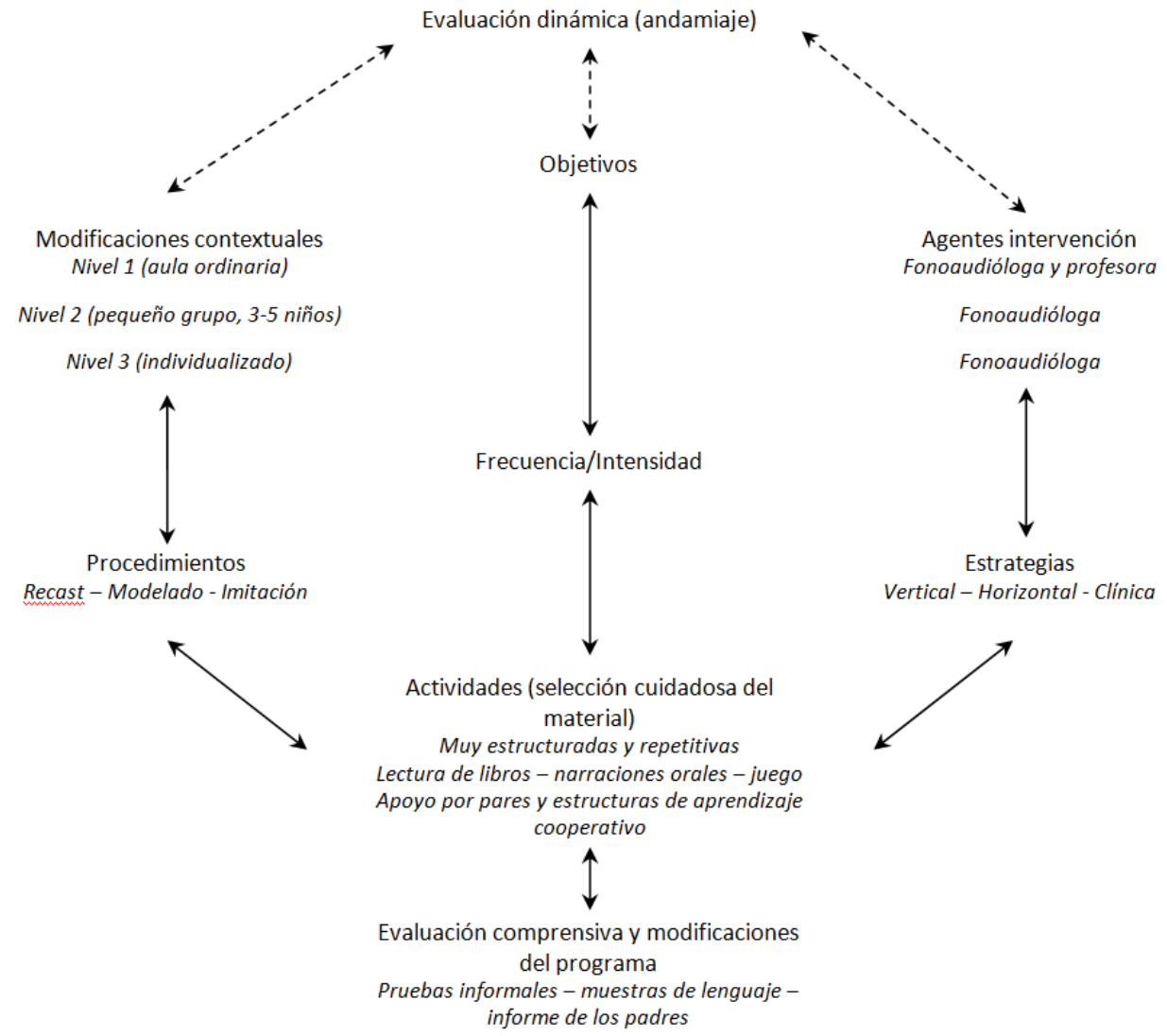

Figura 1. Modelo de intervención para alumnado con TEL (a partir de McCauley y Fey, 2007).

Debido a las limitaciones de espacio del presente manuscrito solo se explicarán los distintos niveles organizativos previstos en este acercamiento.
Se trata de una cuestión central frecuentemente olvidada y que resulta de vital importancia en los modelos preventivos de respuesta a la intervención 
(próximos a los modelos $R T I$ usados en el ámbito anglosajón). De esta forma, en el nivel 1, la profesora, con el asesoramiento y el apoyo de la fonoaudióloga, trabaja con todos los niños en el aula habitual, aunque haciendo un mayor seguimiento de aquellos que presentan un TEL. En este proceso resulta clave la colaboración educativa entre fonoaudiólogas y profesoras porque proporciona a estas últimas los instrumentos, las estrategias y los procedimientos necesarios para asegurar una enseñanza de calidad para todo el alumnado. Se trata, en definitiva, de priorizar una actividad de lenguaje entroncada con el curriculum y que, mediante evaluaciones periódicas, se identifique a los que no progresen adecuadamente dentro del aula ordinaria.

Sin embargo, a veces la intervención con todo el alumnado dentro del aula no es suficiente para cubrir las necesidades de los $\mathrm{TEL}^{59,60}$. En esta situación debe recurrirse a otro tipo de acción más estratégica que permita asegurar la adquisición de habilidades básicas para el desarrollo del lenguaje oral y de la lectura temprana. Con este propósito se activará el nivel 2, organizando a los niños en pequeños grupos (entre dos y cinco alumnos) dentro o fuera del aula ordinaria, en un intento de incrementar el apoyo de los TEL, ofrecido ahora de manera colegiada por la profesora y la fonoaudióloga. Por último, y en aquellos casos en los que el progreso no sea el esperado, se dará paso a una educación diferenciada en el nivel 3; ahora los niños con TEL reciben una enseñanza más explícita, individualizada e intensiva. Esta organización diversificada de intervención ha sido utilizada con éxito para la obtención de habilidades en niños con TEL. En las tablas II y III se muestra un resumen de dichos hallazgos.

Tabla II. Niveles 1 y 2 de intervención.

\begin{tabular}{|c|c|c|c|c|c|}
\hline Habilidad & Actividades & Estrategia & Procedimientos & Niveles 1 y 2 & Bases teóricas \\
\hline $\begin{array}{l}\text {-Pragmática: } \\
\text { conversación } \\
\text {-Producción } \\
\text { narrativa } \\
\text {-Vocabulario } \\
\text {-Habla } \\
\text {-Conciencia } \\
\text { fonológica (nivel } \\
\text { intersilábico) } \\
\text {-Lectura temprana }\end{array}$ & $\begin{array}{l}\text {-Asamblea } \\
\text {-Scripts } \\
\text {-Plan EDEN (Pavez, } \\
\text { Coloma y Maggiolo, } \\
\text { 2008) } \\
\text {-Lectura cuentos } \\
\text {-Definir dibujos } \\
\text {-Clasificar dibujos } \\
\text {-Definición oral } \\
\text {-Discriminación de } \\
\text { sonidos } \\
\text {-Memoria auditiva } \\
\text {-Segmentar palabras } \\
\text { en sílabas } \\
\text {-Lectura dialógica } \\
\text { - Explicar y hacer } \\
\text { predicciones }\end{array}$ & Horizontal & $\begin{array}{l}\text {-Manipulación del } \\
\text { contexto para ofrecer } \\
\text { ocasiones para la } \\
\text { atención conjunta } \\
\text {-Brindar múltiples } \\
\text { oportunidades } \\
\text {-Empleo de } \\
\text { andamiaje, modelado } \\
\text { interactivo y } \\
\text { encadenamiento de } \\
\text { preguntas } \\
\text {-Estructurar, focalizar } \\
\text { y enriquecer el input }\end{array}$ & $\begin{array}{l}\text { Nivel } 1 \text { (grupo } \\
\text { grande, aula } \\
\text { ordinaria) y } \\
\text { Nivel } 2 \text { (grupo } \\
\text { pequeño) }\end{array}$ & $\begin{array}{l}\text {-Teorías del } \\
\text { aprendizaje y del } \\
\text { constructivismo } \\
\text { social. }\end{array}$ \\
\hline
\end{tabular}


Tabla III. Nivel 3 de intervención.

\begin{tabular}{|c|c|c|c|c|c|}
\hline Habilidad & Actividades & Estrategia & Procedimientos & Nivel 3 & Bases teóricas \\
\hline $\begin{array}{l}\text {-Conciencia } \\
\text { fonológica (niveles } \\
\text { intra silábico y } \\
\text { fonémico) } \\
\text {-Habla } \\
\text {-Producción y } \\
\text { comprensión } \\
\text { narrativa } \\
\text {-Morfosintaxis }\end{array}$ & $\begin{array}{l}\text {-Implementación de los } \\
\text { programas Los sonidos } \\
\text { de las Palabras (García, } \\
\text { 2008) y ALE (González y } \\
\text { Cuetos, 2008) } \\
\text {-Producción de } \\
\text { palabras, sílabas y } \\
\text { fonemas, con atención } \\
\text { conjunta y movimientos } \\
\text { corporales } \\
\text {-Grabar cuentos y } \\
\text { escucharlos } \\
\text {-Lectura y recontado de } \\
\text { libros con estructura } \\
\text { narrativa clara (Patata, } \\
\text { tomate y pimiento) } \\
\text {-Conversación sobre } \\
\text { actividades y dibujos } \\
\text { animados; lectura } \\
\text { conjunta de historias. } \\
\text {-Uso de oraciones } \\
\text { coordinadas y } \\
\text { subordinadas }\end{array}$ & Vertical & $\begin{array}{l}\text {-Ofrecimiento de } \\
\text { repeticiones } \\
\text { indirectas como } \\
\text { feedback } \\
\text {-Apoyo contextual } \\
\text { (rutinas) } \\
\text {-Respuestas } \\
\text { contingentes } \\
\text { (atención, interacción } \\
\text { verbal) } \\
\text {-Reducción de la } \\
\text { complejidad de los } \\
\text { modelos lingüísticos } \\
\text { ofrecidos al niño. } \\
\text {-Empleo del } \\
\text { modelado interactivo, } \\
\text { la práctica } \\
\text { estructurada, una } \\
\text { respuesta sistemática } \\
\text { y regular a las } \\
\text { producciones del } \\
\text { niño, y el } \\
\text { reforzamiento. }\end{array}$ & $\begin{array}{l}\text { Nivel } 3 \\
\text { (trabajo } \\
\text { individual, } \\
\text { fuera del aula) }\end{array}$ & $\begin{array}{l}\text {-Modelos de } \\
\text { naturaleza más } \\
\text { conductual } \\
\text {-Modelo } \\
\text { transaccional }\end{array}$ \\
\hline
\end{tabular}

\section{Enriquecimiento psicolingüístico y neuropsicológico en la intervención.}

El modelo de intervención que hemos venido implementando ha logrado resultados positivos en los últimos años (para una revisión se puede consultar Acosta, Moreno y Axpe $^{11,29}$ ), aunque habría que realizar algunas precisiones. Así, y con carácter general, en primer lugar, los niños con TEL progresan ostensiblemente en el aprendizaje del lenguaje y de otras áreas del curriculum (por ejemplo, en la lectura), al mismo tiempo que lo hacen en su desarrollo emocional y social. En segundo lugar, el avance en lenguaje no se da de manera similar en todos sus componentes; esto es, parece haber un mejor desarrollo en habilidades conversacionales, vocabulario y habla que en morfosintaxis, narrativa y procesamiento fonológico. En tercer lugar, se aprecia un ritmo pausado en su evolución circunstancia que puede hacer peligrar el éxito escolar del alumnado con TEL. En consecuencia y antes de emprender un nuevo ciclo de acción, nos planteamos las siguientes cuestiones: ¿por qué el alumnado con TEL no progresa de manera adecuada en todas las áreas del lenguaje sobre las que se interviene de manera frecuente e intensa? ¿Por qué no es capaz de seguir un ritmo adecuado que le permita, con el paso de los años, ponerse a la altura de sus iguales? El modelo es de naturaleza dinámica por lo que ya ha ido implantando algunas modificaciones durante su desarrollo, es decir, se han diversificado los contextos de intervención y se ha trabajado de manera transdisciplinar, se ha combinado el uso de procedimientos, estrategias, actividades y materiales, 
y se ha aumentado la frecuencia e intensidad de las sesiones.

Sin embargo, una nueva propuesta deberá introducir algunos cambios que complementen las bases organizativas, curriculares y fonoaudiológicas de nuestro modelo de intervención. En esa dirección está surgiendo una reciente iniciativa que incorpora al trabajo del alumnado con TEL un atractivo bloque de contenidos destinado a mejorar las habilidades cognitivas y neuropsicológicas, con el propósito de alcanzar un rendimiento óptimo en las diversas funciones ejecutivas, al objeto de maximizar los beneficios de nuestro trabajo.

En efecto, en los últimos años parece ponerse en evidencia algunas características ciertamente deficitarias de naturaleza neuropsicológica que acompañan al TEL, algunas de las cuales ya hemos enumerado anteriormente, pero a las que ciertamente se les ha dedicado poca atención, en los diferentes programas de intervención fonoaudiológica. Más concretamente es preciso hacer referencia a determinados problemas de atención $^{61}$, de alteraciones en la velocidad de procesamiento $^{62}$, de déficit en la memoria de trabajo $^{61,63}$ y de dificultades en la planificación y en la inhibición ${ }^{64}$, todo ello muy conectado con las funciones ejecutivas de estos niños, es decir, con aquellas capacidades mentales necesarias para la formulación de objetivos y la planificación de estrategias idóneas para alcanzar dichos objetivos, optimizando el rendimiento ${ }^{65,66}$. Por lo tanto lo que se propone es la introducción de contenidos de carácter neuropsicológico, relacionados con la enseñanza de determinadas funciones ejecutivas por medio del apoyo en distintos aspectos como pueden ser en las rutinas y en las reglas de la clase, en proporcionar organizadores en forma de claves visuales, en el ofrecimiento de estrategias de organización y de priorización, en reforzar la memoria de trabajo, en ayudar en el mantenimiento y la monitorización de la atención sostenida, en favorecer la habilidad para realizar una correcta planificación de la acción, en manejar el tiempo de forma adecuada y en persistir en la consecución de una meta.

\section{Referencias}

1. Acosta, V., Moreno, A. \& Axpe, A. (2012a). Implicaciones clínicas del diagnóstico diferencial temprano entre retraso de lenguaje y TEL. Universitas Psychologica, 11, 1, 279-291.

2. Conti-Ramsden, G. ( 2011). Aspectos Sociales y Pragmáticos en el TEL: Marcadores diferenciales entre el TEL y los trastornos del espectro autista (TEA). Actas del III Congreso Internacional del Colegio de Logopedas de Cataluña, 3-4.

3. Fey, M. (1986). Language intervention with young children. Boston: Allyn and Bacon.

4. Paul, R. (2007). Language disorders from infancy through adolescence: Assessment and intervention. St. Louis, MO: Mosby Elsevier.

5. Tomblin, J., Records, N. \& Zhang, X. (1996). A system for the diagnosis of specific language impairment in kindergarten children. Journal of Speech, Language and Hearing Research, 39, 1284-1294.

6. Leonard, L., Deevy, P., Miller, C., Charest, M. \& Kurtz, R. (2003). Surface forms and grammatical functions: Past tense and passive participle use by children with specific language impairment. Journal of Speech, Language and Hearing Research, 46, 43-55. 
7. Messer, D. \& Dockrell, J. (2006). Children's naming and word-finding difficulties: Descriptions and explications. Journal of Speech, Language and Hearing Research, 49, 309-324.

8. Redmond, S. (2003). Children's productions the affix ed in past tense and past participle contexts. Journal of Speech, Language and Hearing Research, 46, 10951109.

9. Coady, J. \& Evans, J. (2008). Uses and interpretations of non-word repetition tasks in children with and without specific language impairment (SLI). International Journal of Language and Communicative Disorders, 43, 1-40.

10. Conti-Ramsden, G. (2003). Processing and linguistic markers in young children with specific language impairment (SLI). Journal of Speech, Language and Hearing Research, 46, 1029-1037.

11. Acosta, V., Moreno, A \& Axpe, A. (2012b). La acción inclusiva para la mejora de habilidades de lenguaje oral y de lectura inicial en niños con Trastorno Específico del Lenguaje (TEL). Revista de Educación, 359, 332-356.

12. Axpe, A., Acosta, V. \& Moreno, A. (2012). Intervention Strategies in Preschool Students With Specific Language Impairments. Psicodidáctica, 17, 271-289.

13. Moreno, A., Axpe, A. y Acosta, V. (2012). Efectos de un programa de intervención en el lenguaje sobre el desarrollo del léxico y del procesamiento fonológico en escolares de Educación Infantil con Trastorno Específico del Lenguaje. RIE, 30, 1, 71-86.

14. Ukrainetz, T. \& Gillam, R. (2009). The expressive elaboration of imaginative narratives by children with specific language impairment. Journal of Speech, Language and Hearing Research, 52, 883-898.

15. Plomin, R., Defries, J., McClearn, G. \& McGuffin, P.(2001). Behavioral genetics. Nueva York: Worth.

16. Ullman, M. \& Pierpont, E. (2005). Specific language impairment is not specific to language: the procedural deficit hypothesis. Cortex, 41, 399-433.

17. Montgomery, J. (2005). Effects of input rate and age on the real time language processing of children with specific language impairment. International Journal of Language and Communication Disorders, 1, 177-188.
18. Martínez, L., Palomino, Barbieri, Z. \& Villanueva, P. (2003). Bases genéticas del trastorno específico del lenguaje. Revista Chilena de Fonoaudiología, 4 (1), 37 49.

19. Villanueva, P., Barbieri, Z., Palomino, M. \& Palomino, H. (2008). Alta prevalencia de trastorno específico de lenguaje en isla Robinson Crusoe y probable efecto fundador. Revista Médica de Chile, 136, 186-192.

20. Bosch, L. (2012). Indicadores tempranos de riesgo en el desarrollo del lenguaje. Actas del XXVIII Congreso Internacional de AELFA, 6-15.

21. Acosta, V., León, S. \& Ramos, V. (1998). Dificultades del habla infantil: Un enfoque clínico. Archidona, Málaga: Ediciones Aljibe.

22. Pavez, M., Coloma, C. \& Maggiolo, M. (2008). El desarrollo narrativo en niños. Barcelona: Ars Médica

23. Gopnik, M. (1990). Feature-blind grammar and dysphasia. Nature, 344, 715.Hodson, B. \& Paden, E. (1991). Targeting intelligible speech: A phonological approach to remediation. Austin, TX: Pro-Ed.

24. Bishop, D (2003). The Children's Communication Checklist, Version 2 (CCC-2). Londres: Psychological Corporation.

25. Reichow, B., Doehring, P., Cicchetti, D. \& Volkmar, F.(2011). Evidence-based practices and treatments for children with autism. Nueva York: Springer.

26. Paris, A. \& Paris, S (2003). Assessing narrative comprehension in young children. Reading Research Quarterly, 38, 36-76.

27. Acosta, V., González, N. \& Lorenzo, C. (2011). Un análisis cualitativo de la estructura episódica, los recursos cohesivos y la diversidad léxica en la narrativa de alumnado con Trastorno Específico del Lenguaje. Aloma, 28, 143-160.

28. Acosta, V., Moreno, A., Axpe, M. \& Lorenzo, M. (2010). Apoyo al desarrollo de habilidades narrativas en niños con TEL en contextos inclusivos. Revista de Logopedia, Foniatría y Audiología, 30, 170-184.

29. Acosta, V., Moreno, A. \& Axpe, A. (2012c). Intervención logopédica sobre habilidades narrativas en niños con Trastorno Específico del Lenguaje. Infancia $y$ Aprendizaje, 35, 2, 201-213. 
30. Carballo, G. \& Fresneda, M. (2005). Evaluación e intervención logopédica en el trastorno específico del lenguaje. Revista de Neurología, 41, 73-82.

31. Cardona, M \& Civit, A. (2008). Valoración dinámica de la habilidad narrativa: aplicación en un caso de trastorno específico del lenguaje. Revista de Logopedia, Foniatría y Audiología, 28, 245-260.

32. Coloma, C. (2012). Sintaxis compleja y discurso narrativo en escolares con TEL. Tesis Doctoral. Universidad de Granada. Inédita.

33. Mendoza, E. (2011). Perfiles psicolingüísticos diferenciales en niños con trastorno específico del lenguaje (TEL). Actas del III Congreso Internacional del Colegio de Logopedas de Cataluña, 4-8.

34. Carballo, G. (2012). Guía para la evaluación del TEL: algunas consideraciones. Revista de Logopedia, Foniatría y Audiología, 32, 87-93.

35. Wetherby, A.\& Prizant, B. (2002). Communication and Symbolic Scales Developmental Profil. Baltimore: Paul H. Brookes.

36. Jackson-Maldonado, D, Thal, D., Marchman, V.A., Fenson, L., Newton, T. \& Conboy, B, (2003). Inventario de Desarrollo Comunicativo MacArthur. Adapt. Española de López-Ornat, S., Gallego, C., Gallo, P. Karousou, A., Mariscal, S. \& Martínez, M. (2005). Madrid: Tea Ediciones.

37. Semel, E., Wiig, E.\& Secord, W. (2004) CELF-4 Screening Test. San Antonio, Texas: Pearson.

38. Smith, M., Brady, J. \& Anastasopoulos, L. (2008). Early Language and Literacy Classroom Observation. Baltimore: Paul H. Brookes.

39. Boudreau, D. (2005). Use of a parent questionnaire in emergent and early literacy assessment of preschool children. Language, Speech, and Hearing in Schools, 36, 33-47.

40. DeBruin, A. (2007). Let's read together. ACIRI. Baltimore: Paul H. Brookes.

41. Acosta, V., Moreno A. \& Axpe, A. (2011a). Análisis de las prácticas de lenguaje oral y de lectura inicial en escolares con Trastorno Específico del Lenguaje en contextos de familia y aula. Cultura y Educación, 23, 43-56.
42. Acosta, V. (2012). La intervención logopédica en los trastornos específicos del lenguaje. Revista de Logopedia, Foniatría y Audiología, 32, 67-74.

43. Monfort, M. \& Juárez, A. (1993). Los niños disfásicos. Descripción y tratamiento. Madrid: CEPE.

44. Aguado, G. (1999). Trastorno Específico del Lenguaje. Archidona, Málaga: Ediciones Aljibe.

45. Mendoza, E. (2001). Trastorno Específico del Lenguaje. Madrid: Pirámide.

46. Hodson, B. \& Paden, E. (1991). Targeting intelligible speech: A phonological approach to remediation. Austin, TX: Pro-Ed.

47. Cleave, P. y Fey, M. (1997). Two Approaches to the Facilitation of Grammar in Children With Language Impairments. American Journal of Speech-Language Pathology, 6, 22-32.

48. Culatta, B. (1994). Representation play and story enactments: Formats for language intervention. En J. Duchan, L. Hewitt \& R. Sonnenmeier (Eds.), Pragmatics: From theory to practice (pp. 105-119). Englewood Cliffs, NJ: Prentice Hall.

49. Fujiki, M. \& Brinton, B. (1994). The social competence of children with specific language impairment. En M. Rice \& R. Watkins (Eds.). Language intervention with children with specific language impairment (pp. 123143). Baltimore: Paul H. Brookes.

50. Paul, R. \& Smith, R. (1993). Narrative Skills in 4-YearOlds in Normal, Impaired, and Late-Developing Language. Journal of Speech and Hearing Research, 36, 592-598.

51. Tellen, S. \& Wren, C. (1985). Acquisition of prepositions in language delayed preschoolers: Is intervention effective?. British Journal of Disorders of Communication, 20, 301-309.

52. Connell, P. (1986). Teaching subject hood to language disordered children. Journal of Speech and Hearing Research, 29, 481-492.

53. Connell, P. \& Stone, C. (1992). Morpheme learning of children with specific language impairment under controlled instructional conditions. Journal of Speech and Hearing Research,35, 844-852. 
54. Steele, S. \& Mills, M. (2011). Vocabulary Intervention for school-age children with language impairment. A review of evidence and good practice. Child Language Teaching and Therapy, 27, 354-370.

55. Skibbe, L., Justice, L. \& Bowles, R. (2011). Implementation processes associated with a homebased phonological awareness intervention for children with specific language impairment. International Journal of Speech-Language Pathology, 13, 110-124.

56. Mendoza, E. (2012). La investigación actual en el Trastorno Específico del Lenguaje, Revista de Logopedia, Foniatría y Audiología, 32, 75-86.

57. Acosta, V., Moreno, A., Cámara, M., Coello, A. \& Mesa, J. (2007). Guía de actuaciones educativas en el ámbito de la comunicación y el lenguaje. Tenerife: Consejería de Educación, Cultura y Deportes. Gobierno de Canarias.

58. Acosta, V., Axpe, A. \& Moreno, A. (2011). Intervención en las dificultades del lenguaje desde un modelo preventivo. Cuadernos de Pedagogía, 412, 58-62.

59. Acosta, V., Moreno, A. \& Axpe, A. (2011b). Intervención en la conciencia fonológica en sujetos con TEL en contextos inclusivos: posibilidades y limitaciones. Bordón, 63, 7-20.

60. Acosta, V., Moreno A. \& Axpe, A. (2011c). Oral language and literacy of pupils with specific language impairment (SLI). Cadernos de Comunicacao $e$ linguagem, 2, 35-47.

61. Buiza, J., Adrián, J. \& González, M. (2007). Marcadores neurocognitivos en el trastorno específico del lenguaje. Revista de Neurología, 44, 326-333.

62. Friederici, A. (2006). The neural basis of language development and its impairment. Neuron, 52, 941-952.

63. Montgomery, J.,Magimairaj, B. \& Finney, M. (2010). Working Memory and Specific Language Impairment: An Update on the Relation and Perspectives on Assessment and Treatment. American Journal of Speech-Language Pathology, 78, 19, 78-94.

64. Akshoomoff, N. (2006). Perceptual organization and visual immediate memory in children with specific language impairment. Journal International Neuropsychological Society, 12, 465-474.
65. Lezak, M. (1995). Neuropsychological assessment (3a. ed.). Nueva York: Oxford University Press

66. Trujillo, N.\& Pineda, D. (2008). Función ejecutiva en la investigación de los trastornos del comportamiento del niño y del adolescente. Revista Neuropsicología, Neuropsiquiatría y Neurociencias, 8, 1, 77-94

Agradecimientos: Agradecemos la ayuda recibida del Ministerio de Economía y Competitividad del Gobierno de España. Proyecto de Investigación Funciones ejecutivas y lenguaje en alumnado con TEL. Un modelo de evaluación $e$ intervención con bases psicolingüística y neuropsicológica. Referencia EDU2011-27789 